\title{
EL INTERLUDIO FENOMENOLÓGICO \\ DE WITTGENSTEIN
}

CARLOS H. SOTO

Universidad de Puerto Rico

\section{INTRODUCCIóN}

Poco después de su regreso a la filosofía en el año 1929, Wittgenstein escribió un breve ensayo titulado "Some Remarks on Logical Form" con la intención de leerlo en una sesión de la Sociedad Aristotélica. Lo repudió poco después de haberlo redactado y procedió a presentar una ponencia diferente. Los críticos de Wittgenstein se han hecho de la vista gorda acerca de las ideas expresadas en él; casi unánime es su veredicto de que, puesto que el autor lo rechazó, no es merecedor de atención. Hallo este juicio muy expedito. En primer lugar, "Some Remarks on Logical Form" revela con claridad el estado del pensamiento de su autor en el preciso momento en que comienza a dar expresión formal a su rechazo del Tractatus. De hecho, en él Wittgenstein da voz a ciertas objeciones que luego hacen aparición en otras de sus obras. En segundo lugar, "Some Remarks on Logical Form" revela una de las ideas más importantes que Wittgenstein adopta con el objetivo de remediar algunas de las tesis del Tractatus que ahora halla insatisfactorias. Se trata de una fenomenología. Un dato que merece atención es que este corto ensayo defiende la tesis de que las propiedades ontológicas de los fenómenos de la experiencia inmediata son responsables de ciertas reglas que integran la sintaxis.

Este giro fenomenológico de Wittgenstein es sorprendente. Resulta ser doblemente sorprendente en vista del hecho de que, a pesar de que él rechazó "Some Remarks on Logical Form", años más tarde le vemos defendiendo la fenomenolo- 
gía que se halla anunciada en este ensayo. Asi, por ejemplo, en sus conversaciones con Schlick y con Waismann, en 1930 le hallamos explicando su fenomenología, y Philosophische Bemerkungen contiene el comentario siguiente:

Para poder tratar adecuadamente los colores simples me es precisa una teoría fenomenológica de los colores. ${ }^{1}$

De manera que, a pesar de que Wittgenstein rechazó el en. sayo de 1929, una importantísima idea que hace su aparición originalmente allí perdura en su filosofía durante los años de 1930-33. Esto merece atención. Debemos entender la vena fenomenológica de Wittgenștein si deseamos tener una concepción clara de la gestación de las ideas que han llegado a constituir el segundo período de su pensamiento.

Me propongo estudiar la fenomenología del Wittgenstein de 1929. En la sección il habré de exponer las ideas más fundamentales que la integran. Luego, en la sección III, paso a problematizar la reacción de Wittgenstein cuando, en 1930, Schlick y Waismann lo confrontan con las consecuencias ontológicas de su fenomenología. Mi tesis es que, a pesar de que Wittgenstein niega explícitamente que su fenomenología admita los juicios sintéticos a priori en el sentido de Husserl, jamás logra eliminar sus consecuencias ontológicas.

\section{LA FENOMENOLIGÍA DE 1929}

La modificación más importante que "Some Remarks on Logical Form" ofrece respecto de las doctrinas del Tractatus es el abandono de la tesis de que las proposiciones elementales son independientes entre sí. La discusión de I929 gira en torno de la incompatibilidad de colores. En el Tractatus Wittgenstein había reconocido que era contradictorio decir que un punto en el espacio visual tiene simultáneamente colores diferentes, pero creía que ello era indicio de que una oración acerca de colores en el espacio visual no es elemental;

1 Phìlosophische' Bemerkungen, sección 218. 
que es capaz de ser objeto de análisis. Lo mismo sostiene acerca de cualquier oración sobre los grados de toda otra cualidad. Wittgenstein creía que las aseveraciones de grados podían ser analizadas como una conjunción de oraciones simples que expresan cantidad, junto con cierta cláusula suplementaria; por ejemplo, tal como uno describiría el contenido del bolsillo del pantalón diciendo: "Contiene un papel, una moneda, unas llaves y nada más." En cambio, en 1929 expresa su convencimiento de que es imposible aceptar esto como análisis de las araciones que expresan grados. Supongamos, dice, que la entidad $E$ exhibe cierto brillo. ¿Cómo habremos de analizar la proposición " $E(2) b$ ", que dice que $E$ tiene dos grados de brillantez? Si aseveramos que tan sólo dice " $E(b)$ $\& E(b)$ ", entonces, por simplificación, resulta que sólo estamos diciendo " $E(b) "$. Y si, por otro lado, tratamos de distinguir las unidades de brillo entre sí y decimos " $E(2) b=$ $E\left(b^{\prime}\right) \& E\left(b^{\prime \prime}\right) "$, no hemos contestado por qué, si $E$ tiene el grado $x$ de la propiedad $F$ en el momento $z$, los otros grados de $F$ no pueden ser ejemplificados por $E$ en $z$.

Esto lleva a Wittgenstein a la conclusión de que las oraciones que atribuyen grados a las cualidades no son atómicas, ya que no admiten análisis, a pesar de que sí son elementales. Dice: "Los números deben ser admitidos en la estructura de las proposiciones atómicas" (p. 162). En otras palabras, las proposiciones atómicas admiten funciones de la forma " $x$ tiene el grado $n$ de la propiedad $F$ ", que dará una proposición verdadera tan sólo con un valor de $n$. Como no más de una proposición de esta clase será cierta en un momento dado, Wittgenstein concluye que las proposiciones elementales que exhiben esta forma no son independientes entre sí. Pero el caso es que admitir esto abre la puerta a ciertas complicaciones acerca de la manera en que se han de concebir las oraciones moleculares. Supongamos que " $P$ " representa la proposición "El punto $x$ en el espacio visual es azul ahora" y que $Q$ representa "El punto $x$ en el espacio visual es rojo ahora"; la siguiente sería la tabla veritativa de su conjunción, según las reglas habituales: 


$$
\begin{array}{lll}
P & \& & Q \\
V & V & V \\
V & F & F \\
F & F & V \\
F & F & F
\end{array}
$$

Pero Wittgenstein sostiene que la primera línea representa "una combinación imposible", ya que representa un intento de ubicar dos cosas diferentes allí donde "s6́lo hay lugar para una". Esto no sería el caso si $P$ y $Q$ fuesen analizables en oraciones mutuamente independientes, tal como concebía el Tractatus. Wittgenstein propone en 1929 la siguiente modificación en la tabla veritativa de la conjunción de $P$ y $Q$ :

$$
\begin{array}{ccc}
P & \& & Q \\
--V & -\forall & -V \\
V & F & F \\
F & F & V \\
F & F & F
\end{array}
$$

Naturalmente, esta táctica supone que las reglas que gobiernan la construcción de las tablas veritativas se pueden deber al tema de las oraciones combinadas entre sí. Wittgenstein admite explícitamente esta tesis tan diferente del Tractatus y caracteriza las nuevas reglas del siguiente modo:

Las reglas nos dicen que, en el caso de ciertas proposiciones atómicas ... hay que excluir ciertas combinaciones de $V_{\mathrm{s}}$ y de $F$ s. (p. 153).

En lo sucesivo voy a utilizar la palabra "reglas" para referirme a reglas tales. $\mathrm{Mi}$ intención es utilizar el suscrito para señalar que son diferentes del resto de las reglas sintácticas, por ejemplo de la regla para la construcción de funciones veritativas de un número de argumentos $n$, i.e., $2^{\text {sn }}$. La palabra "reglas", sin suscrito, referirá a reglas de esta clase. 
En varias conversaciones con Schlick y con Waismann en Viena, en 1929 y 1930, Wittgenstein considera el tema de la incompatibilidad de colores en el contexto de las modificaciones que ha hecho en las tesis del Tractatus. Sus comentarios concuerdan perfectamente con lo que se halla expresado en "Some Remarks on Logical Form". Así, por ejemplo, el día de Navidad de 1929, dice lo siguiente:

Yo tenía la concepción de que las proposiciones elementales debían de ser independientes unas de otras. La descripción completa del mundo sería algo así como un producto de las proposiciones elementales, las cuales son en parte positivas, en parte negativas. Aquí me equivoqué, y lo que hay de falso en ello es lo siguiente:

Yo había establecido las reglas para el uso sintáctico de las constantes lógicas, por ejemplo, "p.q" y no había pensado en que estas reglas podían tener algo que ver con la estructura interna de las proposiciones. Lo falso en mi concepción era que yo creía que la sintaxis de las constantes lógicas se podía establecer sin prestar atención a la conexión interna de las proposiciones. Pero no ocurre así. Yo no puedo decir, por ejemplo: En uno y el mismo lugar están los colores rojo y azul a la misma vez. Aquí el producto lógico es inejecutable. Las reglas para las constantes lógicas forman, antes bien, tan sólo una parte de una sintaxis más comprehensiva, de la cual yo todavía no sabía nada por entonces ... Toda proposición está en un sistema de proposiciones que es aplicado a la realidad como un patrón de medida. (Espacio lógico.) (p. 73)

Concluye, tal como había hecho el ensayo de 1929, que las proposiciones elementales que se excluyen entre sí forman sistemas. Por ejemplo, todas las que hablan de colores forman un sistema y todas las que hablan de sonidos forman otro sistema distinto. Wittgenstein toma de nuevo este tema en otra conversación, el 5 de enero de 1930: luego de sostener que cada sistema representa un espacio lógico, pasa a considerar 
explícitamente la cuestión de si cada proposición pertenece a un sistema, es decir, considera la cuestión de si una proposición como, por ejemplo, $F a$, supone otras, i.e., $F b, F c$, etc. Una discusión similar aparece en la página 317 de Philoso. phische Bemerkungen. Su respuesta es que, si dispusiésemos únicamente de $F a$, sería superfluo mencionar " $a$ ", pues bas. taría con " $F$ ". Añade lo siguiente:

En ese caso la proposición no sería compuesta. Pero es esencial que la proposición sea compuesta. (p. 90)

$\mathrm{Si}$ " $F a$ " es una proposición, debe ser el caso que hay " $F b$ ", " $F c$ ", etc., es decir, ". . F" debe ser satisfecha con valores que forman un sistema. Esta nueva posición de Wittgenstein puede elucidarse del siguiente modo: tomemos la función " $x$ es rojo". Podemos escribir "la mesa" en el lugar de $x$, o cualquier otro nombre de un objeto espacial, pero no, por ejemplo, "el ecuador", ya que el ecuador no es capaz de ser rojo ni no rojo. Una circunstancia similar a ésta es el trasfondo de que, a pesar de que puede ser cierto o falso decir "La mesa es rectangular", carece de sentido decir "La virtud es rectangular". Es evidente que Wittgenstein está sosteniendo que el significado de una función no se halla determinado hasta que se especifica el registro de todos los valores que son candidatos a satisfacerla. Ahora bien, ¿cómo es que los valores de una función forman un sistema? ¿Cómo es que una función que es satisfecha por un argumento excluye los otros valores del mismo sistema? La respuesta en "Some Remarks on Logical Form" es que se debe a las reglası que gobiernan las funciones veritativas admisibles entre las proposiciones elementales no independientes entre sí. Al discutir el modo como las descubrimos, Wittgenstein dice explicitamente que sólo lo hacemos mediante la investigación de los "fenómenos en si mismos". "Tales reglas - dice al final del corto ensayo de 1929 - no pueden ser estipuladas hasta que demos con el análisis definitivo de los fenómenos" (p. 171).

Es evidente que la preocupación de Wittgenstein con los 
fenómenos de la experiencia inmediata comienza a adquirir gran relieve en esta etapa de su desarrollo intelectual. Por ejemplo, estipula que hallamos las reglas

inspeccionando el fenómeno que deseamos describir, tratando de ese modo de entender su multiplicidad ... Es decir, sólo podemos arribar a un análisis correcto mediante lo que podría llamarse el análisis ... de los fenómenos en sí mismos, i.e., que es en cierto sentido a posteriori, no conjeturando sobre posibilidades a priori. (p. 163)

Estas líneas significan que los fenómenos revelan la estructura de las proposiciones elementales. Wittgenstein dice que el análisis de los fenómenos ha de proveer

las formas del espacio y del tiempo, juntamente con el complejo [manifold] de objetos espaciales y temporales, con los colores, los sonidos al igual que con sus gradaciones en varias proporciones, ninguno de los cuales podemos captar mediante nuestros habituales medios de expresión. (p. 165)

Apuntemos de paso que el aire kantiano de este pasaje es demasiado pronunciado para poder atribuirse a la casualidad. Nótese que en el original aparece la palabra manifold y que Wittgenstein habla de las "formas" del espacio y del tiempo.

Al final de su artículo (p. 171) Wittgenstein repite que las reglas $_{1}$ no pueden ser formuladas

a menos que hayamos logrado el análisis de los fenómenos.

Afortunadamente, la conexión entre las reglas ${ }_{1}$ y los fenómenos "en sí mismos" se halla expresada en "Some Remarks on Logical Form". Wittgenstein sostiene que, por un lado, 
las reglas ${ }_{1}, y$, por otro lado, los hechos que constituyen el complejo espaciotemporal, i.e., los fenómenos, son mutuamente heterogéneos en el sentido de que nada en la sintaxis garantiza que el lenguaje tenga la misma multiplicidad lógica que los fenómenos. Nada en la configuración sintáctica del lenguaje garantiza que una forma de representación en particular (un sistema de proposiciones) se halle conectada esencialmente con la forma de su espacio lógico. Wittgenstein admite esto explícitamente al proponer su propia tabla veritativa para la conjunción de $P$ y $Q$. Comenta al respecto que las cuatro líneas de la tabla convencional "otorgan a la proposición una mayor multiplicidad lógica que las posibilidades fácticas", ya que admiten la línea "VVV" (p. 170). Añade enseguida que las varias formas de representación hechas posibles por las reglas sintácticas que no son de la misma naturaleza que las reglas $_{1}$

son las normas de nuestro lenguaje con las que proyectamos, de diversas maneras, muchas formas lógicas diferentes. Por tal razón, no podemos extraer conclusión alguna acerca de la verdadera forma del fenómeno descrito considerando el uso de tales normas. (pp. 164-165)

Surge la pregunta de qué es responsable de la selección, de entre las "diversas formas lógicas", de aquélla que representa esencialmente la forma de la realidad. Respuesta: la forma de los fenómenos de la experiencia inmediata en sí mismos. Wittgenstein sostiene que los fenómenos de la experiencia inmediata explican el modo como las oraciones elementales empíricas tocan la realidad; sostiene que la ontología de los fenómenos de la experiencia inmediata provee la forma de su representación. Ilustra esta tesis de la manera siguiente. Si para poder describir un color en el espacio visual le superimponemos un sistema de coordenadas, podríamos representar el color del modo que sigue: 


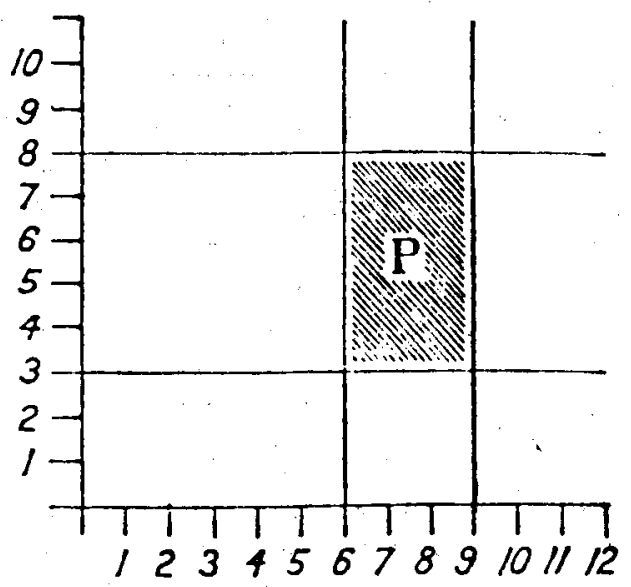

Se desprende que expresaríamos que $P$ es rojo mediante una oración de la forma " $R(6-9,3-8)$ ".

Este mismo ejemplo aparece en 6.341-6.35 del Tractatus con el propósito de ilustrar la primacía del marco lingüístico al describir los hechos. En "Some Remarks on Logical Form" Wittgenstein lo utiliza para recalcar una idea diametralmente opuesta a ésta, a saber, la primacía de los fenómenos descritos en cuanto determinan la red del lenguaje. Naturalmente, la metáfora de la red es tan sólo un recurso retórico, toda vez que no aplicamos red alguna al describir el mundo. Así, por ejemplo, no usamos un red al hablar del pasado; pero en cambio sí usamos fechas, que vienen a ser como los rectángulos de la red en tanto que identifican una porción del campo bajo estudio de suerte que hacen posible aplicar los predicados pertinentes.

Por nuestra cuenta, podemos reconstruir el ejemplo de la red de la siguiente manera. Wittgenstein está admitiendo que algunos de los miembros del lenguaje establecen los objetos de predicación, mientras que otros fijan la aplicación de los predicados. Así, pues, una parte del lenguaje acerca de los objetos cromáticos establece que hablamos de puntos en el espacio visual, mientras que otra parte fija la manera como 
los predicados de la cromaticidad pueden ser aplicados a los puntos en el espacio visual. La primera corresponde a los rectángulos de la red, mientras que la segunda corresponde al procedimiento de colocar la red sobre aquello que se desea describir. Así como cada rectángulo de la red aísla una región en la superficie que es objeto de examen, así mismo podemos concebir que el lenguaje divide el complejo espaciotemporal en segmentos, para cada uno de los cuales hay reglas ${ }_{1}$ que estipulan los diferentes modos de asignarles predicados. $L a$ tesis de Wittgenstein es que el análisis de los fenómenos es

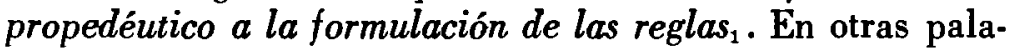

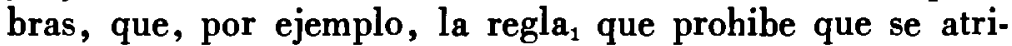
buyan dos predicados cromáticos diferentes a un mismo argumento simultáneamente se debe en última instancia a la naturaleza misma de los fenómenos cromáticos.

Esto lleva a Wittgenstein a sostener, tal como hemos visto, que, de manera contraria a lo que había expresado en el Trac. tatus, las conectivas lógicas no son neutrales respecto de lo que se describe; que su significado se halla capturado en las distintas combinaciones verifuncionales que corresponden a los diferentes campos de objetos espaciotemporales. Tal como ya hemos advertido, en el caso de la conjunción de dos pro. posiciones que expresan cualidades que se excluyen mutuamente, $F F, V F$ y $F V$ representan combinaciones bien formadas, pero no $V V$. ¿Cuál es la naturaleza de esta exclusión? "Some Remarks on Logical Form" contesta de la siguiente manera: aquello que corresponde en la realidad a una función monádica, que contiene constantes espaciotemporales, admite un solo valor. Wittgenstein lo expresa mediante una metáfora:

Aquello que en la realidad corresponde a la función “( ) ET" deja lugar a una sola entidad - en el mismo sentido en que decimos que en una silla sólo hay lugar para una persona. (p. 173)

La exclusión de atributos incompatibles entre sí es repre- 
sentada por las reglas ${ }_{1}$. Ellas prohiben combinaciones tales como " $x$ es azul y rojo simultáneamente". Es por esto por lo que Wittgenstein dice que su formulación supone el análisis de los fenómenos: su formulación, requisito de la aplicación del sistema de prepresentación a la realidad, depende de los fenómenos que expresamos.

Wittgenstein dice que el ejemplo de la red "indica la dirección que debe seguir el análisis de los fenómenos" y pasa a decir lo siguiente acerca de su rol como análisis fenomeno. lógico:

He dicho ... que la proposición "se dirige hacia la realidad", con lo cual quise decir que la forma de los entes se halla contenida en la forma de las proposiciones acerca de ellos. (p. 169)

Wittgenstein ha indicado unas páginas antes que es por medio de los distintos sistemas de proposiciones que las proposiciones "se dirigen hacia la realidad". De tal manera este pasaje significa que el método de prepresentación, ilustrado por la red, ejemplifica el aspecto fenomenológico de la conexión de las reglas ${ }_{1}$ con los hechos. La forma de los sistemas de proposiciones acerca de las entidades cromáticas, por ejemplo, se halla integrada en parte por la regla ${ }_{1}$ "Dos predicados diferentes entre sí no pueden ser atribuidos simultáneamente al mismo nombre de un lugar espacial". Pero ésta es a la vez una indicación de la forma de los entes cromáticos, pues expresa su "forma", expresa implícitamente el registro de combinaciones posibles que los entes cromáticos pueden hacer entre sí. Refleja la naturaleza de éstos al estipular que la aseveración " $x$ aquí ahora" sólo puede tener un valor de entre los diferentes valores que integran el espacio lógico cuyos objetos son candidatos para satisfacerla.

Esto encarna la tesis de que aquello que nos permite hablar y concebir los objetos tal y como lo hacemos es su propia naturaleza. El mismo Wittgenstein pone de relieve que el estudio de los fenómenos no es a priori, sino empírico. Podemos 
hablar de los fenómenos espaciotemporales del modo como lo hacemos porque ésa es la manera como ellos aparecen ante nosotros. Resulta que lo que he llamado reglas ${ }_{1}$, en tanto reflejan esta circunstancia epistemológica, expresan el modo como los objetos fenoménicos se revelan ante nosotros. En efecto, su papel es el de estipular las condiciones necesarias que gobiernan el modo como nos representamos los objetos de la experiencia inmediata. La regla ${ }_{1}$ acerca de los predicados cromáticos gobierna el sistema lingüístico que proyecta los fenómenos cromáticos porque refleja parte de las condiciones necesarias que gobiernan el modo como nos podemos representar los colores.

Es así como el Wittgenstein del 1929 está admitiendo lo que el Tractatus rechazaba, a saber, la necesidad metafísica que gobierna las restricciones de las posibilidades combinatorias de los objetos. Cada objeto - admite el Tractatus en 2.013-2.0131 - se halla en un espacio de estados de cosa posibles: los objetos visuales se hallan rodeados de un espacio visual; las notas musicales, de un espacio auditivo, etc. Un objeto espacial no puede darse fuera del espacio; una nota musical no puede tener ningún color; un olor no puede ser sonoro. ¿Cuál es la naturaleza de esta limitación? ¿Qué necesidad evita que un color tenga un olor? ¿Por qué es que una nota musical carece de dureza? El Tractatus supone que toda necesidad es de índole lógica, de tal modo que estas restricciones necesarias escapan al registro de lo contemplado por la tesis de que toda necesidad se debe a las leyes que regulan el lenguaje. El Wittgenstein de "Some Remarks on Logical Form" viene a reconocer que hay necesidades que no se deben a la naturaleza de los conectivas verifuncionales; reconoce que hay una necesidad metafísica, a saber, la contrapartida fáctica de las reglas ${ }_{1}:$

III. ¿SON LAS REglas 1 SINTÉticas A PRIORI?

1. La vacilación de Wittgenstein

Wittgenstein explica todo esto a Schlick y a Waismann 
a fines de 1929 y principios de 1930. Schlick advierte de inmediato que hay que aclarar si lo que he llamado reglas son únicamente de naturaleza sintáctica, es decir, metalingüística, o si también dependen de la experiencia. Formula la siguiente pregunta: supongamos que una persona ha pasado toda su vida en una habitación de color rojo; ¿puede aseverar que hay otros colores además del rojo? La respuesta de Wittgenstein es que, o bien su sintaxis es idéntica a la nuestra, en cuyo caso la persona posee nuestro mismo sistema de colores, o bien su lenguaje se halla gobernado por una sintaxis diferente de la nuestra, en cuyo caso la persona no se halla familiarizada con ninguno de los colores que nosotros conocemos. Podemos conjeturar que la intención de Wittgenstein es sostener que si la palabra "rojo" de la persona del ejemplo de Schlick tiene el mismo significado de nuestra palabra "rojo", entonces debe estar gobernada por las mismas reglas. Tal como parece ser su costumbre en el transcurso de estas conversaciones, él hace uso de analogías kantianas: una persona que nunca ha salido de una habitación sabe, no obstante, que existe un espacio allende las paredes, y sabe por lo tanto que es posible salir de la habitación. Wittgenstein añade: "Esto no se debe a la experiencia, sino que se halla incluido en la sintaxis del espacio, a priori" (p. 65).

Tal parece que ni Wittgenstein ni Schlick quedaron satisfechos con esta respuesta y regresan al mismo tema unos días más tarde. Schlick pregunta:

$¿$ De qué modo puedo saber que tales y cuales reglas sintácticas imperan? ¿De qué manera sé que el azul y el rojo no pueden estar en el mismo sitio a la misma vez? ¿No es ésta una forma de conocimiento empírico?

Sorprendentemente, Wittgenstein no se dirige a este problema explícitamente. Replica que la respuesta depende del significado de "empírico". Esta respuesta no es satisfactoria. 0 bien Wittgenstein no está en condiciones que afrontar el problema abiertamente, o bien no lo ha advertido. El problema 
es que, al sostener la fenomenología que aparece en "Some Remarks on Logical Form", está admitiendo una clase de oraciones que no son tautológicas a pesar de que sí son necesarias; a saber, las que he llamado reglas ${ }_{1}$, que gobiernan el modo como los diferentes sistemas de proposiciones conectan con sus respectivos espacios lógicos. Posiblemente por lo inaceptable de la respuesta de Wittgenstein, Schlick plantea el problema nuevamente, señalando en esta ocasión que Hus. serl había llegado a pensar que las reglas ${ }_{1}$ eran sintéticas $a$ priori. Wittgenstein rechaza esta posición de Husserl, diciendo que la posibilidad expresada en ellas no es de re. Pero esto tampoco satisface. El objetivo de la pregunta de Schlick es que Wittgenstein diga explícitamente aquello que su fenomenología admite implícitamente, a saber, el rol ontológico de las reglas $_{1}$. En "Some Remarks on Logical Form" es evidente que sí lo tienen. Pero ahora, en 1930, luego de rechazar este ensayo, Wittgenstein parece querer desviar la atención de tal aspecto de su pensamiento. No confirma lo que el ensayo de 1929 a claras luces admite. Pero tampoco lo niega. Meramente repite que las reglas ${ }_{1}$ son sintácticas. Ahora bien, esto nunca ha estado en cuestión, y sin embargo Wittgenstein se refugia en la noción de sintaxis cada vez que surge el tema de la naturaleza ontológica de las reglas ${ }_{1}$. El concepto de sintaxis ya había aparecido en el Tractatus en el contexto de la Teoría de la Mostración: Wittgenstein sostuvo que "la naturaleza de los signos" habla por sí sola (6.124) y por lo tanto las reglas sintácticas se hacen evidentes en los signos (3.334), es decir, se muestran en su empleo. Pero en 1929 y 1930 Wittgenstein sostiene que las proposiciones que tratan la proyección de los hechos en el leguaje son igual de válidas como vá. lidas son las reglas que gobiernan las conectivas verifuncionales.

Adoptando la Tesis de la Mostración, Schlick pregunta si

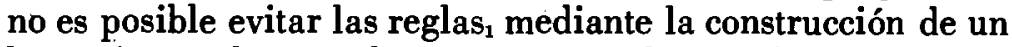
lenguaje en el que sólo se muestran. Siguiendo esta misma estrategia, Waismann dice lo siguiente: 
Si un sistema de signos tiene la multiplicidad correcta, entonces las reglas sintácticas resultan superfluas. El uso de las palabras "al norte de" depende de ciertas reglas sintácticas. No puedo decir " $A$ se halla al norte de $A$ ". Pero los mapas no pueden decir este sinsentido porque ya tienen la multiplicidad adecuada. (p. 70)

Este giro de la conversación es de interés, porque ofrece a Wittgenstein la oportunidad de rechazar explícitamente la tesis de que las reglas ${ }_{1}$ son sintéticas a priori, si así se desea hacer. La Tesis de la Mostración, recuérdese, es una alternativa a la Teoría de los Tipos de Bertrand Russell, de manera que Schlick y Waismannn están ofreciendo a Wittgenstein la oportunidad de rechazar las ramificaciones metafísicas de la Teoría de los Tipos que Wittgenstein admite al adoptar el enfoque metalingüístico de ésta, juntamente con la adopción de una fenomenología.

Wittgenstein no rechaza la deseabilidad de una sintaxis explícita. No acepta la sugerencia tractariana de Schlick y de Waismann. Es evidente que ya no siente antipatía por la Teoría de los Tipos, y de hecho en Philosophische Bemerkungen dice que su concepto de gramática es una teoría de tipos. ${ }^{2}$ Opina, como reacción a la sugerencia de Schlick y de Waismann, que las reglas ${ }_{1}$ son más importantes que las reglas que gobiernan las funciones veritativas ( $\mathrm{pp} .74$ y 80). Su interés recae cada vez más en aquella área de la sintaxis que según él gobierna la construcción de los sistemas de proposiciones, las reglas $_{1}$ : Llega a decir que las reglas de las conectivas verifuncionales deben ser complementadas con reglas ${ }_{1}$ que - afirma él- "forman parte del método de representar el mundo" ( $p$. 81) y que emanan de "la sintaxis interna de las proposiciones" (p. 80).

Evidentemente, esto es lo que preocupa a Schlick. Si las reglas $_{1}$ forman parte del "método de representar el mundo" y derivan de la forma de los entes, entonces el metalenguaje

2 Sección 7. 
que admite el Wittgenstein de 1929-1930 se halla integrado en su parte esencial por reglas que reflejan la constitución de la realidad; se halla integrado por reglas que reflejan las propiedades más generales del mundo fenoménico. Así que en verdad Wittgenstein no ha eliminado la secuela metafísica de su fenomenología en las conversaciones con Schlick y con Waismann, a pesar de que uno parece detectar cierta renuencia a aceptarla abiertamente. Bien mirado, no parece haber tanta diferencia entre la posición de Wittgenstein y la noción de gramática de Husserl en Investigaciones Lógicas.

\section{La ramificación ontológica de las reglas}

Es importante recordar que "Some Remarks on Logical Form" sostiene que las reglas ${ }_{1}$ son el resultado de la forma de los fenómenos. Excluyen ciertas formas de representación, es decir, ciertos sistemas de proposiciones, ciertos modos de enlazar las proposiciones con los hechos espaciotemporales, porque reflejan ciertas imposibilidades fenoménicas. En cambio, admiten otras formas de representación, es decir, ciertos sistemas de proposiciones, ciertos modos de enlazar las proposiciones con los hechos, porque surgen de lo que es posible en el campo de los hechos. Es en esta vena que comienza el ensayo de 1929. Wittgenstein ofrece un breve recuento de algunas de las tesis básicas del Tractatus y pasa a reclamar que la filosofía debe descubrir las proposiciones elementales y formular un simbolismo idóneo para expresarlas, porque el lenguaje cotidiano disfraza la verdadera estructura lógica de las proposiciones y permite la formación de pseudoproposiciones. Toda vez que la sintaxis del lenguaje cotidiano no sirve para evitar estas consecuencias poco deseables, requerimos un "simbolismo adecuado" para poder expresar aquello que en el lenguaje cotidiano es objeto de malinterpretación. El concepto de "simbolismo adecuado" o sintaxis lógica supone que la forma verdadera de las proposiciones no se revela a primera vista y que aquello que se revela a primera vista no sirve para evitar ciertas combinaciones no deseadas. El sim- 
bolismo adecuado tiene como objetivo estipular las restricciones pertinentes, restricciones que descubrimos al estudiar los fenómenos:

Allí donde el lenguaje disfraza la estructura lógica, allí donde permite la formación de pseudoproposiciones ... lo debemos sustituir por un simbolismo que refleje fiel: mente la estructura lógica, que excluya las pseudoproposiciones y que use sus términqs de manera no ambigua. Sólo podemos sustituir el simbolismo inadecuado por uno adecuado por medio de la inspección de los fenómenos que deseamos describir .... sólo llegamos a un análisis adecuado [de los fenómenos] mediante la investigación de los fenómenos en sí mismos. (p. 163)

Los siguientes pasajes expresan el trasfondo de la idea de que descubrimos las reglas ${ }_{1}$ al estudiar los fenómenos; ambos aparecen en las notas de Waismann:

La forma de las proposiciones elementales ... se ajusta a la forma de los fenómenos. (p. 259)

Aquello que se halla descrito por las proposiciones elementales son fenómenos (experiencias). (p. 249)

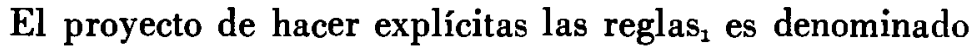
el proyecto de un "Jenguaje Fenomenológico" o "Lenguaje Primario". Wittgenstein así lo informa a Schlick y a Waismann (p. 46) y su definición aparece en la misma primera página de Philosophische Bemerkungen. El Lenguaje Fenomenológico refleja las restricciones esenciales que regulan las posibilidades de combinación de los objetos del complejo espaciotemporal. A tales restricciones Wittgenstein da varios nombres: la esencia del mundo, las propiedades internas de los objetos, la forma de los fenómenos. Únicamente aquello que se constituye de acuerdo con ellas puede ser un fenómeno de la experiencia inmediata.

Como consecuencia de ello, la regla ${ }_{1}$ "Dos predicados cro- 
máticos incompatibles entre sí no pueden ser satisfechos simultáneamente por un mismo argumento" expresa parte de la esencia del mundo; da voz a una de las necesidades que gobiernan a los constituyentes de los fenómenos de la cromaticidad. A pesar de que Wittgenstein no lo admite explícitamente, hay en todo esto cierto eco de lo que alguna vez se ha llamado "síntesis a priori".

En otras palabras, en 1929-1930, Wittgenstein ha acudido al campo de la experiencia inmediata como manantial de donde surjen las reglas ${ }_{1}$. Su Lenguaje Fenomenológico revela las condiciones de la experiencia del mundo, de donde emanan las reglas. Éstas, pues, se deben al acceso extralingüístico que Wittgenstein parece suponer que tenemos de la realidad. De la estructura que descubrimos gracias a esta facultad extralingüística nacen las restricciones que incorporamos en la sintaxis para que el lenguaje resulte tener la adecuación esencial requerida para poder reflejar la realidad fielmente. ${ }^{3}$

BIBLIOGRAFIA

1. Ludwig Wittgenstein, "Some Remarks on Logical Form", Proceedings of the Aristotelian Society, 1929.

2. - Philosophische Bemerkungen, Franckfurt: Suhrkamp Verlag, 1964.

3. F. Waismann, Ludwig Wittgenstein und der Wiener Kreis, Oxford: Blackwell, 1967.

3 Deseo agradecer las valiosas sugerencias de George Wilson y de Román Castilla Lázaro. 
A study of Wittgenstein's "Some Remarks on Logical Form" is done from the point of view of the phenomenology it espouses. A brief presentation is made of the main points of his concern with the phenomena of immediate experience as that which validates the rules of meaning. This view is compared with that of Husserl. A survey is made of Wittgenstein's defense of his Phenomenology as it is registered by $\mathrm{F}$. Waismann. It is held that Wittgenstein flirts with the synthetic a priori. 